\title{
EVALUACIÓN Y SOPORTABILIDAD DE LOS BOFEDALES DE LA CUENCA DEL UCHUSUMA ZONA ALTOANDINA DE TACNA - 2006
}

Responsable : MVZ. Cecilio Hurtado Quispe. Miembro : MVZ. Cesario Cruz Anchapuri.

\section{RESUMEN}

El presente articulo tiene como objetivo evaluar la cobertura vegetal y la composición vegetal, la producción de biomasa de los bofedales altoandinos de Tacna. Se utilizó el método de "transección al paso"; y para el censo agrostológico se empleó el muestreador de "Estructuras de puntos"; los resultados obtenidos fueron de $58,82 \%$ de cobertura vegetal y $41,18 \%$ sin valor forrajero, con una composición botánica de 36 especies vegetales y una producción de biomasa húmeda de $885,80 \mathrm{~kg} . \mathrm{M}$.H./ha y biomasa seca de 279,23 kg.M.S./ha. Se estimó una soportabilidad para MV de 221,23 UAL/año y 347,05 UAL/año para MS y una capacidad de carga de 0,43 UAL/ha/año MV y 0.69 UAL/ha/año MS.; El proceso de desertificación, provocado por la derivación de las aguas superficiales y subterráneas, ha roto el equilibrio entre suelo, vegetación y agua, causando pérdida de especies vegetales, baja producción de biomasa forrajera, degradación de la calidad de pastura y pérdida de peso vivo.

\section{ABSTRACT}

The object of the study was to evaluate the vegetable coverage, the vegetable composition, and the production of biomass of the high Andes marshes of Tacna. The "Step Transplanting" method was used for the agricultural census, and the "Point structures" sampling was put to use. The results obtained were $58.82 \%$ vegetable coverage and $41.18 \%$ without foraging value with a botanical composition of 36 sorts of vegetables and a humid production of biomass of $885.80 \mathrm{k} . \mathrm{g} . \mathrm{M} . \mathrm{H}$./ha and dry biomass of $279.23 \mathrm{k.g.M} . \mathrm{S}$./ha. It was estimated that the supporting limits of green material was 221.23 UAL every year and 347.05 UAL/yr for dry material with a holding capacity of $0.43 \mathrm{UAL} / \mathrm{ha} / \mathrm{yr}$ for green material, and 0.69 UAL/ha/yr for dry material; the desertification process, provoked by the derivation of superficial and subterranean waters, has broken the equilibrium between earth vegetation and water, causing a loss of vegetable species, low foraging biomass production, degradation of the pastures, and the loss of living weight.

\section{INTRODUCCIÓN}

El trabajo de investigación se realizó en la cuenca del río Uchusuma ubicada en los CPM de Alto Perú y Ancomarca, pertenecientes al distrito de Palca, departamento de Tacna. El estudio consistió en evaluar la cobertura vegetal, la composición vegetal y la producción de biomasa de los bofedales altoandinos.

El sobrepastoreo es el principal problema de los humedales o bofedales que reduce la cobertura vegetal. Por otra parte, los bofedales presentan varios problemas como: mala circulación del agua, salinización y actividades antropogénicas (sistemas de trasvase de agua). El objetivo es evaluar la cobertura vegetal, la composición vegetal, la producción de biomasa y estimar la soportabilidad y capacidad de carga de los bofedales. El estudio realizado se considera trascendente porque ayudará, entre otros aspectos, a conocer la evaluación y soportabilidad de los bofedales de la cuenca del Uchusuma de la zona altoandina de Tacna, que en la actualidad carece de todo tipo de estudio en esta zona. Por otra parte, la investigación servirá para contrastar con otros estudios realizados en regiones colindantes, que permitirá realizar comparaciones sobre la situación de los bofedales.

\section{MATERIALY MÉTODOS}

\section{Ubicación del estudio}

La investigación se realizó en la microcuenca del río
Uchusuma ubicada en los CPM de Alto Perú y Ancomarca, pertenecientes al Distrito de Palca, Región de Tacna, se encuentra entre los 3000 y $5700 \mathrm{msnm}$, entre las coordenadas $17^{\circ} 30^{\prime}$ a $17^{\circ} 40^{\prime}$ de latitud Sur y $69^{\circ} 30^{\prime}$ a $69^{\circ}$ $50^{\prime}$ de longitud Oeste, se extiende hasta la frontera con Chile y Bolivia.

El objetivo del estudio es evaluar la cobertura vegetal, la composición vegetal, y la producción de biomasa y estimar la soportabilidad, capacidad de carga de los bofedales altoandinos de Tacna.

\section{Metodología}

Determinación de la Cobertura y Composición Vegetal: Los humedales o bofedales se pueden evaluar mediante diversas técnicas agrostológicas con la finalidad de medir sus atributos. Una de las técnicas aplicadas para las condiciones de nuestra zona altoandina es el método de "transección al paso", que sirve para conocer la soportabilidad de los pastizales y el empleo de la técnica de cosecha de biomasa verde por metro cuadrado, ello nos permite conocer el número de animales que se puede pastorear en una determinada área de pastizal.

Materiales Utilizados: mapa del área de estudio, estructura de puntos, para determinar la frecuencia de especies, wincha metálica, registros de evaluación.

Transección al Paso: permitió hacer un inventario de las especies vegetales, determinar la cobertura vegetal, conocer 
las especies deseables, poco deseables e indeseables, determinar el grado de erosión del suelo y juzgar la condición del pastizal.

Procedimiento de la Evaluación de los Bofedales: a) Reconocimiento del pastizal; b) Censo agrostológico de los bofedales; c) Análisis de registro de campo, Determinación de índices; e) Condición del pastizal.

Estimación de la Producción de Biomasa Forrajera: La determinación de la producción de biomasa se expresa en valores de biomasa y rendimiento forrajero de la comunidad vegetal del bofedal, permitiendo conocer la actividad real que ofrecen estos sistemas como fuente alimenticia de importancia altoandina.

Materiales Utilizados: para determinar la cobertura vegetal, se utilizó un cuadrante metálico de $25 \times 25 \mathrm{~cm}$. $(0,0625 \mathrm{~m} 2)$, balanza de precisión, bolsas de papel, hoz, cuchillo y registro de campo.

\section{Procedimiento para Determinar la Biomasa Verde: a)} Muestreo de la vegetación b) Determinación de muestras c) Cosecha de la muestra vegetal d) Cálculos de rendimiento de materia verde de pastizal.

Capacidad de Carga Animal y Soportabilidad: a) Cálculo de capacidad de carga animal: Capacidad de carga animal = Rendimiento de pasto por hectárea / consumo de pasto/animal/periodo; b) Cálculo de soportabilidad: Soportabilidad $=$ Rendimiento de pasto*área de sitio/consumo de pasto/animal/periodo de pastoreo.

\section{RESULTADOS Y DISCUSIÓN}

\section{Composición Florística y Cobertura Vegetal:}

En el Cuadro $N^{\circ} 01$ se muestra la cobertura y la composición florística, de Paucarani (testigo); de las evaluaciones realizadas se identificaron 19 especies vegetales predominantes con una cobertura vegetal de $72,06 \%$ y un $27,94 \%$, sin valor forrajero; también se determinaron las principales especies floristicas dominantes hasta la tercera especie, siendo como primera especie Calamagrostis minima $(31,71 \%)$, como segunda especie Oxychloe andina (19,38\%), como tercera especie Carex ecuadorica $(16,30 \%)$; y los indicadores de palatabilidad son de $26,98 \%$ de especies deseables, $30,79 \%$ de especies poco deseables y $14,29 \%$ de especies no deseables.

Cuadro $\mathrm{N}^{\circ}$ 01: Composición floristica y cobertura.

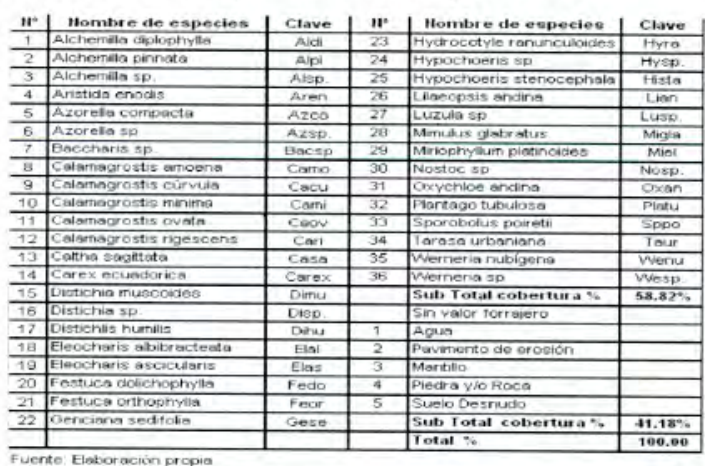

En el cuadro $N^{0} 01$ también se observa los resultados generales obtenidos en la zona de estudio, se identificaron 36 especies vegetales predominantes con una cobertura vegetal de $58,82 \%$ y $41,18 \%$ sin valor forrajero; se determinaron las principales especies floristicas dominantes hasta la tercera especie, siendo como primera especie Eleocharis ascicularis $(15,72 \%)$, como segunda especie Distichia sp (14,25\%) y como tercera especie Calamagrostis minima $(11,46 \%)$ (Fig. 01); también se observa el porcentaje de palatabilidad de $36,08 \%$ de especies deseables, $14,79 \%$ de especies poco deseables y $7,95 \%$ de especies no deseables.

Según el análisis de los resultados encontrados para la zona de estudio por diferentes investigadores, se identificaron 36 especies vegetales. Sin embargo, nuestro hallazgos muestran divergencia con otros. Asi, Condori E, et al. (2001) reportó en Puno para la provincia del Collao 45 especies vegetales, presentó una elevada composición florística con respecto a nuestro estudio; para Tarata se reportaron 21 especies vegetales, ampliamente divergentes con nuestro resultado. En Bolivia, Prieto G. et al. (2001) reportó 58 especies vegetales en bofedales altoandinos; estos resultados nos indican que en el ámbito de nuestro estudio existe una menor distribución de especies frente al sistema TDPS Boliviano. Este resultado confirma que en nuestra ámbito de estudio existe la desaparición de especies en los bofedales. La cobertura vegetal es de $58,82 \%$ y $41,18 \% \sin$ valor forrajero para nuestro estudio; sin embargo nuestros hallazgos muestran divergencia y similitudes con otros. Condori E, et al. (2001) reportó en Puno para la provincia del Collao una cobertura promedio de $94,59 \%$ y el $5,41 \%$ sin valor forrajeo y para Tarata se reportó una cobertura vegetal de $95,65 \%$ y $4,35 \%$ sin valor forrajero. En Bolivia, Prieto G. et al. (2001) reportó $76,00 \%$ para los bofedales mésicos alcalinos, $3 \%$ para los bofedales mésico neutros.

En Chile, Olivares (1987) reportó para el ecosistema bofedal altoandino una cobertura vegetal de $50 \%$ a $100 \%$. En Tacna, ONERN (1987) reportó una cobertura vegetal para la asociación vegetal Distichetum entre 80 y 100\%. Como podemos observar, nuestros hallazgos muestran divergencias muy preocupantes debido a las diferencias encontradas entre uno y otro resultado, en algunos casos hasta alcanzan un ciento por ciento. Esto nos hace inferir que existen efectos ambientales producidos por el "Proyecto Vilavilanil Etapa" sobre este ecosistema.

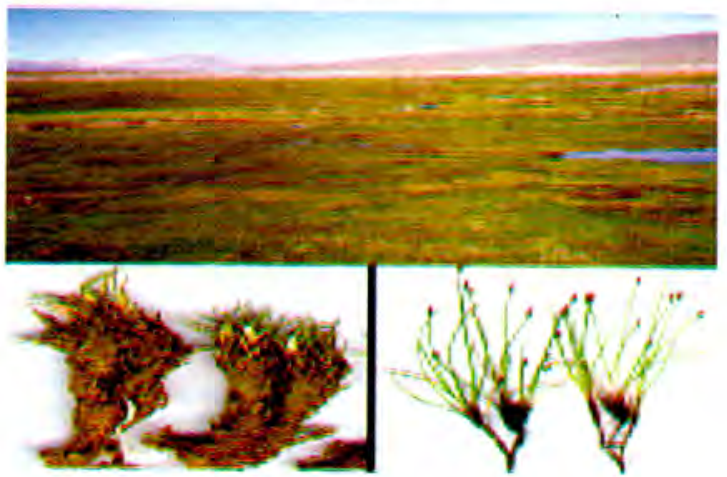

Figura $\mathbf{N}^{0}$ 01: Cobertura vegetal y las dos principales especies dominantes Eleocharis ascicularisy Distichia sp 


\section{Producción de Biomasa de los Bofedales de la Cuenca delUchusuma:}

Cuadro N 02: Producción de biomasa de los bofedales de la cuenca del Uchusuma.

\begin{tabular}{|l|r|r|c|}
\hline \multirow{2}{*}{ Lugar o Sectores } & \multicolumn{2}{|c|}{ Biomasa } & \\
\cline { 2 - 4 } & Humeda & \multicolumn{1}{c|}{ Seca } & Condición \\
\cline { 2 - 4 } & KgM.V/ha & KgM.S/ha & Pastizal \\
\hline Calaparque, Charaque, Alto Perú & 1696,60 & 493,22 & Regular \\
\hline Choraque Chapi, Rosaspata & 85,50 & 43,50 & Muy pobre \\
\hline Viluyo, Parco, Ticuta & 549,87 & 264,53 & Pobre \\
\hline Hospicio, Chontacollo, Vilapuraca & 1211,54 & 315,67 & Buena \\
\hline Total Biomasa & 885,80 & 279,23 & \\
\hline Testigo & 2123,60 & 702,13 & Regular \\
\hline Paucarani
\end{tabular}

\section{Fuente: Elaboración propia}

En el Cuadro $\mathrm{N}^{\circ} 02$ se consigna el resultado de Paucarani (testigo), presenta una producción de biomasa húmeda de 2123,60 kgM.V./ha, y una producción de biomasa seca de $702,13 \mathrm{~kg}$. M.S. /ha, condición de pastizal regular.

En el Cuadro $\mathrm{N}^{\circ} 02$ se aprecia la determinación general de la producción de biomasa para la zona de estudio, que es de 885,80 kg.M.H./hay 279,23 kg.M.S./ha.

Otros estudios realizados por INIATECHNOSERVE (2000) mencionados por Condori $E$, et al. (2001): para el Collao obtuvieron producciones completamente superiores para materia verde de $8,669,80 \mathrm{kgM}$.V./ha para Sullkanaca, $6165,60 \mathrm{kgM} . V$./ha para San José y 10875,70 kgM.V./ha para Jihuaña; mientras que para materia seca fueron de 3163,63 $\mathrm{kgM}$.S./ha para Sullkanaca, 2726,15 kgM.S./ha para San José y 2978,35 kgM.S./ha para Jihuaña.

Resultados obtenidos para la provincia de Tarata Tacna, fueron de 8406,52 kgM.V./ha y 2469,29 kgM.V./ha; éstos resultados presentan diferencias superiores en rendimiento de producción de biomasa en estas comunidades alpaqueras. Estos hallazgos confirman que la producción de biomasa en nuestra zona de estudio es menor debido a poca oferta forrajera, como posibles efectos del proyecto Vilavilani I Etapa, en la regulación del almacenamiento de agua por la laguna de Paucarani.

Estudios realizados en Puno por Condori E, etal. (2001), reportaron para la provincia del Collao materia verde de $7332,60 \mathrm{kgM} . V$./ha a más de $25000 \mathrm{kgM} . \mathrm{V}$./ha, teniendo como promedio $12957,98 \mathrm{kgM} . \mathrm{V}$./ha, promedios en materia seca $3724,14 \mathrm{kgM} . \mathrm{S} . / \mathrm{ha}$ y $4119,29 \mathrm{kgM} . \mathrm{S}$./ha. Comparativamente con los resultados obtenidos en la zona de estudio, la producción general de biomasa es de 885,80 kg.M.H./ha y 279,23 kg.M.S./ha. Como se puede observar en los resultados, el rendimiento de producción de biomasa verde y seca es completamente superior en la provincia del Collao en materia verde y seca; mientras que el rendimiento de la producción es muy bajo para la zona de estudio en materia verde y seca.

Trabajos realizados en Bolivia por Prieto G. et al. (2001) reportaron un promedio general de rendimiento de biomasa de $4508,8 \mathrm{kgM} . \mathrm{S} / \mathrm{ha}$ por separado en el piso ecológico altoandino, los bofedales mésico neutros $3213,6 \mathrm{kgM}$.S/Ha y mésicos alcalinos con $1196 \mathrm{kgM} . \mathrm{S} . / \mathrm{Ha}$, estos hallazgos confirman los rendimientos superiores de biomasa verde y seca con relación a la Microcuenca del Uchusuma alcanzando $885,80 \mathrm{~kg} . \mathrm{M} . \mathrm{H}$./ha y $279,23 \mathrm{~kg} . \mathrm{M} . \mathrm{S}$ esto demuestra que se viene produciendo una degradación de bofedales incontenible por falta de agua.

Otros estudios realizados en Bolivia en Ulla Ulla por Villarroel (1997) indican que las especies Hypochoeris taraxacoides y Distichia muscoides son muy palatables, de mayor presencia en bofedales en época húmeda, y expresan rendimientos de materia verde $1705,2 \mathrm{kgM}$.V./ha y 1635,5 kgM.V./ha. Alzérreca (1986) para la ecorregión de puna semiárida y árida reporta $2540 \mathrm{kgM}$.S./ha con dominancia de Oxychloesp.

Estudios realizados en Chile, Parinacota por Castellano et al. (1998), obtuvieron una disponibilidad de materia seca de $1382 \mathrm{kgM} . S$./ha a $3089 \mathrm{kgM} . S$./ha, entre la época seca y lluviosa; estos hallazgos confirman los rendimientos superiores de biomasa verde y seca frente a la zona de estudio, alcanzando $885,80 \mathrm{~kg}$. M.H./ha y $279,23 \mathrm{~kg}$. M.S; esto demuestra que se viene produciendo una degradación de bofedales por falta de agua.

\section{Capacidad de Carga Animal y Soportabilidad:}

Cuadro $\mathrm{N}^{\circ}$ 03: Capacidad de carga y soportabilidad de los humedales o bofedales

\begin{tabular}{|c|c|c|c|c|}
\hline \multirow{3}{*}{ Lugar o Sectores } & \multicolumn{2}{|c|}{ Capacidad de Carga } & \multicolumn{2}{|c|}{ Soportabilidad } \\
\hline & M. Verde & M. Seca & M. Verde & M. Seca \\
\hline & UAL/ha/año & UAL/ha/año & UAL/ha/año & UAL/halaño \\
\hline Charaque, Calaparque, Alto Perú & 0,84 & 1,23 & 566,63 & 823,84 \\
\hline Choraque, Chapi, Rosaspata & 0,04 & 0,11 & 14,41 & 36,81 \\
\hline Viluyo, Parco, Ticuta & 0,27 & 0,66 & 119,3 & 287,03 \\
\hline Hospicio, Chontacollo, Vilapuraca & 0,6 & 0,79 & 184,58 & 240,53 \\
\hline Total & 0,43 & 0,69 & 221,23 & 347,05 \\
\hline \multicolumn{5}{|l|}{ Testigo } \\
\hline Paucarani & 1,06 & 1,75 & 331,41 & 548,01 \\
\hline
\end{tabular}

Fuente: Elaboración propia

En el Cuadro $N^{\circ} 03$ se muestra los resultados obtenidos en Paucarani (testigo). Se reportó una capacidad de carga animal de 1,06 UAL/ha/año para materia verde y 1,75 UAL/ha/año para materia seca; una soportabilidad de 331,41 UAL/año para materia verde y 548,01 UAL/año para materia seca, en condición de pastizal regular.

En el Cuadro $N^{\circ} 03$ también se aprecia la determinación de la capacidad de carga y la soportabilidad para la zona de

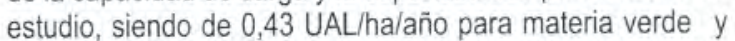
$0,69 \mathrm{UAL} /$ ha/año para materia seca; mientras que la soportabilidad para materia verde es 221,23 UAL/año y 347,05 UAL/año para materia seca.

Según estudios realizados en Puno por INIATECHNOSERVE (2000) mencionado por Condori E, et al., (2001) encontró en puna seca una capacidad de carga de 2,5 UAA/ha/año para Sullkanaca, 2,8 UAA/ha/año para el sector San José y 2,0 UAA/ha/año para Jihuaña; estos hallazgos confirman una mayor capacidad de carga animal en estas comunidades alpaqueras, frente a una menor capacidad encontrado en la zona de estudio. Como consecuencia, existe una menor producción de biomasa ante la disminución del caudal de agua del río Uchusuma por regulación por la laguna de Paucarani. 
Estudios realizados en Bolivia por Luna D, (2001) encontraron que la capacidad de carga en las unidades administrativas de Ulla Ulla-La paz en puna seca oscila entre 0,2 y 2,2 UAL/ha/año.

Los bofedales hidromórficos son asociaciones con mayor capacidad de carga de 0,7 a 2,2 UAL/ha/año; estos hallazgos son similares a los encontrados en la zona de estudio que es de 0,43 UAL/ha/año para materia verde y 0,69 UAL/ha/año para materia seca; mientras que la soportabilidad para materia verde es 221,23 UAL/año y 347,05 UAL/año para materia seca.

Estudios realizados por ONERN (1987) reportaron en el inventario y evaluación agrostológica de la zona altoandina de Tacna, la presencia de 4,500 ha con una capacidad de carga de 2,0 U.Alaño dado por su alto valor agrostológico compuesto por asociaciones, tales como la Distichia muscoides, con una soportabilidad de 9000 alpacas. Asimismo existen pastizales que ocupan 8,585 ha con capacidad de carga de 1,0 U.Avaño, estos pastizales está representando por la asociación Calamafgrostis cephalanta que, a su vez, soporta 8585 alpacas.

También existen asociaciones vegetales tales como la Parastrephia lepidophilla. Festuca ortophylla y Calamagrostis breviaristada, abarcando una extensión de 193,950 ha, con capacidad de carga de 0,33 U.Alaño, con una soportabilidad de 64,003 alpacas.

Finalmente, existe la presencia de asociaciones muy pobres, tales como Parastrephia lepidophylla Calamagrostis breviaristada, Margiricarpus strictus Festucha dichaclada, cubriendo una extensión de 32,350 ha con una soportabilidad de 0,17 U.A/año, llegando a recibir 5,499 alpacas; estos hallazgos son similares con nuestro estudio de 0,43 UAL/ha/año para materia verde, y 0,69 UAL/ha/año para materia seca; mientras que la soportabilidad para materia verde es 221,23 UAL/año y 347,05 UAL/año para materia seca.

\section{CONCLUSIONES}

1. Se determinó la cobertura vegetal de $58,82 \%$ y $41,18 \%$ sin valor forrajero, con una composición botánica de 36 especies vegetales y una producción de biomasa húmeda de $885,80 \mathrm{~kg} . \mathrm{M} . \mathrm{H}$./ha y biomasa seca de $279,23 \mathrm{~kg}$. M.S. $/ \mathrm{ha}$.

2. Se estimó la soportabilidad para materia verde siendo de 221,23 UAL/año y 347,05 UAL/año para materia seca y una capacidad de carga de $0,43 \mathrm{UAL} / \mathrm{ha} /$ año para materia verde y $0,69 \mathrm{UAL} / \mathrm{ha} / \mathrm{año}$ para materia seca.

\section{BIBLIOGRAFÍA}

Condori E. Choquehuanca D. 2001. Evaluación de las caracteristicas y distribución de los bofedales en el ámbito peruano del sistema TDPS. Subcontrato 21.12. Universidad Nacional del Altiplano Puno, Facultad de Ciencias Biológicas. 1era ed. Editorial Talleres de la UNA. Puno, Perú.

Farfan R. Durant A. 1998. Manejo y técnicas de evaluaciones de pastizales altoandinos. Publicaciones Técnica, FMV Nº 39, Nov 1998. Estación Experimental Marangani LaRaya. Cusco.

Grupo Tragsa. 2003. La Ingenieria en los procesos de desertificación. 1era ed., Ediciones Mundi-Prensa Castelló. 37. 28001, Madrid.

Instituto de Investigación y Promoción de Camélidos Sudamericanos. 1992. Programa de Desarrollo Integral de Camélidos Sudamericanos Micro Región Tarata, Tacna. Convenio Micro Región Tarata Universidad Nacional del Altiplano, Puno, Facultad de Medicina Veterinaria y Zootecnia. 1era ed. Editorial Talleres de la U.N.A. Puno, Perú.

Luna D, Alzérreca H. Prieto G. Céspdes J. 2001. Estudio de capacidad de carga de los bofedales para la cría de alpacas en el sistema T.D.P.S. Bolivia. 1era ed. Editorial Plural Editores. LaPaz.

Malpartida E. 1990. Pautas de manejo de las praderas naturales en la zona alpaquera. Informe Técnico № 16. Serie: pastos. Proyecto Alpacas INIAA. Puno, Perú.

Oscanoa L. 1988. Diagnóstico de los recursos naturales y capacidad de carga de los pastizales en el sector Cclacocha y el Fundo Munaypaata. Informe técnico No 01. Proyecto Alpacas COTESU/IC. Puno, Perú.

Prieto G. Alzérreca H. Laura J. Luna D, Laguna S. 2001. Características y distribución de los bofedales en el ámbito boliviano del sistema T.D.P.S. 1era ed. Editorial Plural Editores. La Paz.

Rocha O. Sáez, 2003. Uso pastoril en humedales altoandinos. 1era ed. Editorial Plural Editores. LaPaz. 